let $a_{i}$ be the exponent denoting the highest power of $p_{i}$ still dividing $n, a_{i}>0$. Further, let the function $f(x)$ mean the number of all existing primes $\leq x$. Putting $f\left(p_{i}\right)-f\left(p_{i+1}\right)=b_{i}(i<m), f\left(p_{m}\right)=b_{m}$, we can use the second or third method above to store the numbers $a_{1}, b_{1}, \cdots, a_{m}, b_{m}$. These determine the factorization completely.

Stig Comét

Matematikmaskinnämnden

(The Swedish Board for Computing Machinery)

Box 6131, Stockholm 6, Sweden

1. R. L. Bivins, N. Metropolis, P. R. Stein, \& M. B. Wells, "Characters of the symmetric groups of degree 15 and 16," $M T A C$, v. 8, 1954, p. 212.

2. S. CoMÉ, "On the machine calculation of characters of the symmetric group," Tolfte Skand. Matematikerkongressen, Lund, 1953, Comptes rendus, p. 18-23.

3. G. H. HARDY \& S. RAMANUJAN, "Asymptotic formulae in combinatory analysis," London Math. Soc., Proc., s. 2, v. 17, 1918, p. 75.

4. A series of eight papers by A. Young, "On quantitative substitutional analysis," published in London Math. Soc., Proc., beginning in ser. 1, v. 32, 1901, p. 384, and ending in ser. 2, v. 37, 1934 , p. 441.

5. T. NAKAYAMA, "On some modular properties of irreducible representations of a symmetric group," Jap. J. Math., v. 17, 1941, p. 165 and p. 411.

6 . This involves shifting to the left until the most significant digit is 1 , and counting the shifts; see G. KJELLBERG \& G. NEOvius, "The BARK, a Swedish general purpose relay computer," $M T A C$, v. 5,1951 , p. $29-34$.

\title{
Determination of Steiner Triple Systems of Order 15
}

1. Introduction. A Steiner Triple System is a set of subsets of $n$ marks such that: a) Each subset contains three distinct marks, b) each pair of distinct marks appears in one and only one subset. It is easy to see that $n \equiv 1$ or $3(\bmod 6)$ [1] and that the number of subsets is $n(n-1) / 6$. For $n=3,7,9$ there is one and essentially only one such set, all others being obtained by permutation of the marks. For $n=13$, there are two distinct systems.

Using SWAC for the computations we have obtained the non-isomorphic systems of order 15. During the course of the calculations, Prof. L. J. PAIGE called our attention to a series of papers [2] which culminate in the listing by F. N. Cole of a set of 80 systems of order 15 with proof that these are distinct and an argument tending to show that the listing is exhaustive. Since following the argument would entail tremendous hand calculation and since the number 80 was below our expectation so that it seemed quite possible that there had been errors or omissions in the determination, we decided to continue. It turns out that these 80 are indeed complete and that Cole's computations were either correct or at any rate did not contain any errors which led to the omission of a valid system. In any case these results testify to Cole's reputation for brilliant computation.

2. The computation-General considerations. There are two stages in the calculation, the formation of the systems and the reduction of isomorphisms. That these must be carried out simultaneously is seen from the fact that if all systems on 15 marks which arise from the 80 distinct systems are counted, the total exceeds $6.10^{13}$ (actually (14521/315)15!). 
In practice the reduction was carried out at three places. First the total number of systems to be computed by SWAC was reduced to the order of $10^{4}$ by giving the machine partial systems to complete in such a way that all distinct systems were certain to be included in the final result. Second, in the later stages of the computation it was possible to inhibit output of computed systems by a simple test for isomorphism to previously determined systems which could be added to the completion routine. Finally the output systems had to be tested for permutations taking them into other outputs.

3. Initial data. It is clear that, given any Steiner Triple System on fifteen marks, by renaming we may assume that the triples $(1,2,3)(1,4,5)(1,6,7)$ $(1,8,9)(1,10,11)(1,12,13)(1,14,15)(2,4,6)$ are present. It is then easy to see that there are essentially only four ways to complete the triples containing 2 . These are:

$$
\begin{array}{ll}
\text { A : } & (2,5,7)(2,8,10)(2,9,11)(2,12,14)(2,13,15) \\
\text { B : } & (2,5,7)(2,8,15)(2,9,10)(2,11,12)(2,13,14) \\
\text { C: } & (2,5,8)(2,7,9)(2,10,12)(2,11,14)(2,13,15) \\
\text { D : } & (2,5,8)(2,7,10)(2,9,12)(2,11,14)(2,13,15) .
\end{array}
$$

Cole calls these respective arrangements, triple tetrad, single tetrad, hexad, and duodecad, since the first has three sets of four triples in the form: $(1, \alpha, \beta)$ $(1, \gamma, \delta)(2, \alpha, \gamma)(2, \beta, \delta)$. The second has only one such tetrad and the third has two sets of the form $(1, \alpha, \beta)(1, \gamma, \delta)(1, \epsilon, \zeta)(2, \alpha, \gamma)(2, \beta, \epsilon)(2, \delta, \zeta)$. D has no combination of interlocking triples short of the full subsystem with the obvious omission of $(1,2,3)$.

Again, it is apparent that, in case A, we may assume the presence of either $(3,4,7)$ or $(3,4,8)$. For suppose the triple $(3,4, k), k>8$; there is a set of four triples $(1, k, j)(1, m, n)(2, k, m)(2, n, j)$; the permutation $(8, k)(9, j)(10, m)(11, n)$ will produce the triple (348) leaving the remainder invariant. Further, in the case (347) we may assume the remaining triples including 3 to be $(3,5,6),(3,8,11)$ $(3,9,10)(3,12,15)(3,13,14)$ for if, say, $(3,5,8)$ occurred, the permutation (45) (67) will leave the triples on 1 and 2 invariant while introducing (348).

In a similar fashion the cases considered under $\mathrm{B}, \mathrm{C}$, and $\mathrm{D}$, may be reduced. In this reduction the automorphism group on the partial system plays an important role. Finally, we may consider the sets in a hierarchy. Under B, no system which includes triple tetrads on the triples using any pair of marks $(a, b)$ to replace $(1,2)$ in the definition of triple tetrad above need be considered since a permutation of the form $(1, a)(2, b) \cdots$ would reduce it to one of the A systems. Under $\mathrm{C}$ no system with a tetrad comes into consideration. Under D we can rule out any system containing either a hexad or a tetrad. This consideration actually serves to exclude $\mathrm{D}$ completely.

The length to which this procedure is carried must be balanced between several conflicting requirements :

1) The machine should produce as few systems as possible.

2) There should be as few starts as possible since each new subsystem demands $\mathrm{ne}$ feeding of initial data which is time-consuming to prepare. 
3) The initial hand computation must not be carried to a point where there is danger of losing completeness by errors arising from its complexity.

The requirement 3 ) is less important than it appears at first glance since it is far easier to neglect existing automorphisms (which would create over-production) than to introduce non-existing ones. At any rate 2) and 3) together determined that no production of initial data beyond triples on 3 was considered. The initial starts, then, included from 14 to 19 fixed triples. For example, in the case A: (348) the following additional triples were used : 1 . $(3,5,6), 2 .(3,5,9)(3,12,15)$ $(3,13,14), 3$. $(3,5,10)(3,12,15)(3,13,14), 4 .(3,6,12)$. On start 1 , the machine was stopped after $(3,7,9),(3,7,10),(3,7,11)$, had been considered.

4. Exhaustive production of Steiner Triple Systems. As remarked the computations were performed on SWAC. This is a binary machine using 36 digits plus sign in a word. The high speed memory consists of 256 words and the medium speed memory was a drum holding 4096 words. These sizes limited the choice of routines and the methods of storage.

The machine is required to form all systems using the given first triples. Rather than a list of triples the machine actually stores and works with an incidence or usage table. Specifically, if we designate the first four 8 digit portions of a SWAC word by $\alpha, \beta, \gamma, \delta$, each mark is stored in $\alpha$ of a corresponding word; $\beta$ holds the number of marks with which the given mark has appeared at that stage of the computation; the $i$ th position of the first fifteen digits of $\gamma$ and $\delta$ holds a 1 if the given mark has been used with $i$ and a 0 otherwise. Thus the cell corresponding to the mark 8 in the entry A: (348) case, initially read in decimal: $\alpha=008, \beta=007, \gamma=241, \delta=192$. Note that an incidence of a mark with itself is always counted so as to instruct the machine unambiguously that a mark $i$ is available for use in a triple with a mark $j$ if and only if a 0 appears in the $i$ th place of $(\gamma, \delta)$ in the word corresponding to $j$. With this and the binary representations of decimal $241(1111,0001)$ and decimal $192(1100,0000)$, it is immediately seen that the triples $(1,8,9)(2,8,10)(3,4,8)$ generate the line.

The basic code may be outlined as follows:

\begin{tabular}{|c|c|c|c|c|c|}
\hline Routine & $\begin{array}{c}\text { Possible } \\
\text { Pre- } \\
\text { decessors }\end{array}$ & Operations Performed & $\begin{array}{l}\text { Successor } \\
\text { Criterion }\end{array}$ & Yes & No \\
\hline A & $\mathrm{F}$ & Find least $\alpha$ whose $\beta<15$ & Are all $\beta \geq 15$ ? & B & $\mathrm{D}$ \\
\hline B & A & $\begin{array}{l}\text { Output (or go to supple- } \\
\text { mentary routine for } \\
\text { search for earlier iso- } \\
\text { morphs) }\end{array}$ & $\begin{array}{l}\text { No choice except } \\
\text { possibility of in- } \\
\text { serting supple- } \\
\text { mentary routine }\end{array}$ & $\mathrm{C}$ & $\mathrm{C}$ \\
\hline $\mathrm{C}$ & $\begin{array}{l}\text { B or supple- } \\
\text { mentary } \\
\text { routine }\end{array}$ & $\begin{array}{l}\text { Return to next earlier } \\
\text { value of } \alpha\end{array}$ & No choice & $\mathrm{D}$ & $\mathrm{D}$ \\
\hline $\mathrm{D}$ & $A$ or $C$ & $\begin{array}{l}\text { Determine marks avail- } \\
\text { able for use with pres- } \\
\text { ent choice of } \alpha \text { value }\end{array}$ & No choice & $\mathrm{E}$ & $\mathrm{E}$ \\
\hline
\end{tabular}


DETERMINATION OF STEINER TRIPLE SYSTEMS OF ORDER 15

Possible

Pre-

Routine decessors

E

D

F

G

H

I

E

E

G

H
Operations Performed

Permute to form new subsystem on mark denoted by present value of $\alpha$

Store new subsystem with other completed ones

Have all permutations for this $\alpha$ been tried? its list
Successor

\section{Criterion}

Yes No

Is a new subsystem $\quad F \quad G$ found?

A A

H E tions for this $\alpha$ been tried?

E I

Is an earlier $\alpha$

G
available? 
The permutation scheme of routine $\mathrm{E}$ is a modification of a Paige-Tompkins routine [3] adapted to the fact that order within a triple is of no importance and thus there are only $(n-1)(n-3) \cdots 1$ permutations on $n$ marks to be tried. Both the permutation scheme and the complete code can be illustrated by the example A: (348) (356). Here 1 and 2 are complete, their $\beta$ is 15 . For 3, 4, 5, 6, 8, $\beta=7$. For $7,9,10,11,12,13,14,15, \beta=5$. Thus 3 is selected as the least number still incomplete with largest possible $\beta$. The numbers 7,$9 ; 10,11 ; 12,13 ; 14,15$; are available for use with 3 , the semicolons indicating the initial breakdown into pairs which could form triples with 3. Permutation occurs as far to the left as possible, either because of impossible pairings or fully utilized sub-systems. Here $(10,11)$ is the first impossible one. The next considered is 7,$9 ; 10,12 ; 13,14$; 15,11 . This is acceptable and the lines of the table are stored in this order on a drum channel; the permutation tallies are also stored for future reference. The incidence tables are then adjusted in the high speed memory to account for this sub-system and we proceed. This time 4 is selected with its list of 7,$9 ; 10,11$; 12,$13 ; 14,15$. The successive trials from this point to form a new sub-system are: 1$) 7,10 ; 11,12 ; 13,14 ; 15,9 ; 2) 7,10 ; 11,12 ; 13,15 ; 9,14 ; 3) 7,10 ; 11,12$; $13,9 ; 14,15 ; 4) 7,10 ; 11,12 ; 14,15 ; 9,12 ; 5) 7,10 ; 11,13 ; 14,9 ; 12,15$. The italics indicate the (first) impossible pairing. Of course, after step 3 ) the order returns momentarily to 1 ) while the tallies are adjusted to indicate completion of this level and to start the permutation at the next level in the prescribed pattern.

After storage, 7 is called with 8,$11 ; 12,13 ; 14,15$ associated. This time no acceptable sub-system can be found so 4 is recalled and begins permuting where it left off. It happens that this entry on 3 cannot be completed; none of the twenty apparently acceptable sub-systems on 4 associated with it lead to a complete system. Not until $3 ; 7,11 ; 12,15 ; 9,10 ; 13,14$ does a completable entry on 3 arise.

To output, the $\alpha$ entries from the successive storage drum channels are collected in a compact arrangement and printed or punched. The code described here was written by LAVERNE E. RICKARD with suggestions from C. B. TOMPKIns and the authors.

5. Reduction to distinct systems. For the A and B levels the problem was run with no additional routine. Using a tabulation of the output, those systems which were obviously isomorphic to earlier systems but had been generated because it was simpler to let the machine run than to cut them out by increasing the number of starts (requirement 2 above) were discarded by hand.

In the $\mathrm{C}$ computation, a routine was added before output. This routine looked at each completed system checking possible tetrad linkages. If one was found the output was by-passed and the next system was computed. This resulted in the production of only two C-systems which were found to be isomorphic on hand-checking.

The problem of removing the non-obvious isomorphisms still remained. At this point some 5000 systems were left in the running. It was manifestly impossible to try any considerable proportion of the 15 ! permutations on the possible pairs of isomorphic systems. Accordingly, using a modification of a procedure of Louise D. Cummings contained in the cited memoir, a set of invariants was tabulated for each system.

Given a triple system, the sub-system consisting of the triples on any mark possesses one of the linkage types A, B, C, D, with the sub-system on each of 
the 14 other marks. To each mark, then, can be correlated a vector $(a, b, c, d)$ where $a+b+c+d=14 ; a$ is the number of A linkages with the given mark, $b$ the number of $B$ linkages, etc. Thus associated with each system is a $15 \times 4$ matrix and if two systems are isomorphic their matrices are row permutations of each other. Further, if system $S_{1}$ is carried into system $S_{2}$ by the permutation $\left(\begin{array}{llll}1 & 2 & \cdots & 15 \\ i_{1} & i_{2} & & i_{15}\end{array}\right)$, the vector corresponding to $j$ of $S_{1}$ must be equal to the vector corresponding to $i_{j}$ of $S_{2}$. A routine was written for SWAC which computed these vectors (and their sum for sorting purposes) for each system. When a system was input, a table was first formed which stored in the $(i, j)$ position the number $k$ appearing in the triple $(i, j, k)$ of the system. Zero was stored in the $(i, i)$ positions. Since $4 \cdot 15=60$ digits were required for any line of the table, double precision computation was necessary. The main part of the routine then took each ordered pair $(i, j)$ and found the coincidences. If there were three tetrads a tally was added in the $\alpha$ section of the $i$ th output word, etc. In working with the $B$ systems a rejection step was added whenever a triple tetrad occurred.

There now remained 1912 A-systems and 538 B-systems which were sorted into stacks according to the equivalent matrices. The final routine used a representative of each of the stacks and attempted to find a permutation taking the successive systems into this archetype; i.e., choices were made for $i_{1}$ and $i_{2}$ from the possible numbers with correct associated vectors. This determined $i_{3}$ from $(1,2,3)$ which was checked for vector equality. If it did not check, a new choice was made for $i_{2}$; if it did check, a choice for $i_{4}$ was made which determined $i_{5}$ from $(1,4,5), i_{6}$ from $(2,4,6), i_{k}$ from $(34 k), i_{m}$ from $(35 m)$, etc. Each of these was checked for vector equality and for consistency. (E.g., if $m=6, i_{6}=i_{m}$.) The permutation found was then output. It turned out that the invariants were complete in the sense that all systems with equivalent matrices were isomorphic.

6. Time factors and controls. Systems were produced at rates varying from 20 per minute down to three per minute depending on the relative "richness" of the initial starts. The linkage computation required about 2 seconds per system. The final permutation routine required an average of 4 seconds per system including typewriter output of the permutation.

All routines used were essentially self-checking. In the exhaustive production routine the possibility of a consistent error in all the usage tables is negligible. An error will show up as " $i$ is available for use with $j$ but $j$ is not available with $i$." When this happened the machine went into a short loop and could only be restarted by feeding consistent data. Several hand computational checks were made to insure that it was indeed producing the "next system." It may be noted that every possible distinct system occurred in at least two isomorphs. Some of them occurred 200 times.

The checks on the vector computation were that the matrix obtained was symmetric, that every vector had component sum 14 , and that the matrices were consistent with others of the same type.

For the permutation routine, the various requirements of consistency were again such as to make the possibility of finding a false permutation negligible. An error could, however, cause an answer of "not isomorphic" to appear. This did, in fact, happen in several cases. In each case the system was re-fed twice; the resulting permutations were compared and hand checked. 
7. Conclusions. The chief source of wasted time lay in the lack of initial knowledge concerning the magnitude of the problem:

a) The original output of the Rickard code was in converted decimal, designed for easy tabulation and hand procedures. These outputs had to be reconverted into binary form readily assimilated by SWAC.

b) Systems with A-linkage should have been rejected without output during the B-computation as was done for tetrad linkage in the C-computation. In fact, the linkage vectors should have been computed simultaneously with the system. However, we had not thought of these matrices until these calculations were complete.

The procedures described in this paper represent, to the best of our knowledge, the first use of large scale digital computing equipment to isolate isomorphic algebraic systems. In this connection, the crucial step is the devising of a reasonably calculable system of invariants to reduce the gross search for mappings. By its nature, a computer is better adapted to calculating such invariants than to exhaustive search for transformations.

The question of computing the systems of order 19 arises. Here there are seven possible starts on the systems using 2 ; these may be represented by the possible partitions of 16 into even numbers greater than 2 in the same way that $A, B, C, D$, in the fifteen case correspond to $4+4+4,4+8,6+6,12$ respectively. For each of these starts there are $2 \cdot 10^{6} a$ priori distinct permutations of $(4,5, \cdots, 19)$ for use with 3 compared to $10^{4}$ on $(4,5, \cdots, 15)$. Machine procedures would be required to isolate the starts and the total number requiring trial might be as great as $10^{4}$. The problem thus seems to be beyond the reach of present equipment. Some short runs of systems have been made on SWAC and these bear out this impression. Unfortunately, no reliable estimate of the total number of systems to be expected exists.

The authors wish to acknowledge the assistance given them in coding and computation by C. B. Tompkins, L. J. Paige, Ruth B. Horgan, LaVerne E. Rickard, and John Selfridge. The codes used are on file at Numerical Analysis Research, University of California, Los Angeles.

Marshall Hall, Jr.

J. D. SwifT

Ohio State University

University of California, Los Angeles, and Institute for Advanced Study

The computations on SWAC were sponsored by the Office of Naval Research and the Office of Ordnance Research. The preparation of this paper has been sponsored in part by ONR Contract NR 045141.

1. It has been known since 1859 that this necessary condition is also sufficient for the existence of a Steiner triple system. M. REIss, "Ueber eine Steinersche combinatorische Aufgabe," J. Reine Angew. Math., v. 56, 1859 , p. 326-344.

2. A. S. White, F. N. Cole, \& Louise D. Cummings, Complete Classification of Triad Systems on Fifteen Elements, Memoirs of the National Academy of Sciences, v. XIV, 1925, Second Memoir, $89 \mathrm{p}$.

3. C. Tomprins, "Machine attacks whose variables are permutations," Amer. Math. Soc. Sixth Symposium on Applied Math. Proceedings, McGraw-Hill (in press).

4. R. A. FISHER, "An examination of the different possible solutions of a problem in incomplete blocks," Annals of Eugenics, v. 10, 1940, p. 52-75. (Lists 79 systems; the system of Type C, Cole's "Heffter System" was missed.) 